\title{
Utilidad de la malla de polietileno de baja densidad, en la plastia inguinal sin tensión.
}

\author{
Eduardo Escobedo ${ }^{1}$, David Esmer-Sánches ${ }^{1}$ y Juan Francisco Hernández-Sierra ${ }^{2}$ \\ ${ }^{1}$ Departamento de Cirugía, Hospital Central Ignnácio Morones Prieto. San Luis de Potosí, \\ México. \\ ${ }^{2}$ Departamento de Posgrado, Facultad de Medicina,Universidad de San Luis de Potosí, \\ México.
}

Palabras clave: hernia inguinal; plastia inguinal; malla de polietileno.

Resumen. La malla de polietileno de baja densidad (PEBD) tipo mosquitero, se ha utilizado como una alternativa barata a la de polipropileno (PP) para la reparación de hernias inguinales sin tensión. El objetivo fue comparar ambas en términos de dolor, infección, rechazo y recurrencia. Se realizaron 31 plastias inguinales con técnica de Liechtenstein en 28 pacientes, utilizando en forma aleatoria 1) malla de PEBD ó 2) malla PP. Se registró en forma ciega infección y dolor en el postoperatorio, a los 7 y 30 días, y rechazo o recurrencia a 3, 6 y 18 meses. No existieron diferencias significativas entre los grupos de PEBD y PP, en dolor, infección, rechazo o recurrencia. El costo por procedimiento fue 58\% menor en el grupo de PEBD. Se concluye que la malla de PEBD es una alternativa segura y económica para la plastia inguinal abierta sin tensión ya que es 500 veces más económica que la de polipropileno. 


\title{
Utility of a low-density polyethylene mesh in inguinal hernia repair without tension.
}

\author{
Invest Clin 2018; 59 (4): 318 - 324
}

Key words: inguinal hernia; hernia repair; polyethylene mesh.

\begin{abstract}
The low-density polyethylene (LDPE) mesh mosquito-net type has been used as an economical alternative to the polypropylene (PP) mesh for the repair of inguinal hernias without tension. The objective of this work was to compare both materials in terms of resulting pain, infection, rejection and recurrence of the hernia. We performed 31 inguinal hernioplasties with the Lichtenstein technique in 28 patients, randomly using the 1) LDPE mesh or the 2) PP mesh. Postoperative infection and pain were recorded at seven and 30 days, and rejection or recurrence at three, six and 18 months. There were no statistically significant differences between the groups of LDPE and PP, with respect to infection, recurrence and rejection frequency. Measured pain was similar in both groups. The cost per procedure in the LDPE group was 58\% lower compared to the PP group. We concluded that the properties of the LDPE mesh are similar to those of the PP mesh. There were no differences in infection, rejection and recurrence, and LDPE mesh is 500 times cheaper than the polypropylene mesh.
\end{abstract}

Recibido 02-06-2018 Aceptado 13-12-2018

\section{INTRODUCCIÓN}

La incidencia de hernia inguinal es de aproximadamente 5 a $10 \%$ de la población general. Es 8 veces más frecuente en el sexo masculino, por lo que los hombres requieren 20 veces más cirugías, por esta causa, que las mujeres $(1,2)$.

Liechtenstein perfeccionó la técnica abierta de reparación quirúrgica con malla sin tensión en 1984 y desde entonces se convirtió en el procedimiento de elección en el mundo para la reparación de hernias inguinales. Los pacientes presentan menor dolor postoperatorio y regresan más temprano a la actividad normal, con una menor tasa de recurrencia (3).

El reto actual de la cirugía es disminuir costos en los procedimientos, realizando cirugías ambulatorias con anestesia local de rápida recuperación. Sin embargoo, no se ha logrado disminuir el costo de las prótesis o mallas. El costo de una malla puede llegar a representar hasta un $40 \%$ del costo total del procedimiento (4). Las mallas de baja densidad "tipo mosquitero", han sido evaluadas con diferentes resultados $(5,6)$, por lo que el objetivo de este trabajo fue evaluar la eficacia reportada en términos de dolor mediante la escala visual análoga (EVA), infección del sitio operatorio, rechazo y recurrencia, de la malla de polietileno de baja densidad (PEBD) en comparación con la malla de polipropileno (PP), en la plastia inguinal abierta con malla sin tensión, en un país con condiciones socio-económicas y sanitarias diferentes a países desarrollados.

\section{PACIENTES Y MÉTODOS}

El estudio se realizó entre abril y diciembre de 2017 e incluyó 28 pacientes con diagnnóstico de hernia inguinal directa o indirecta, unilateral o bilateral. Los criterios de inclusión fueron: edad entre 16 y 75 años, de cualquier sexo, que aceptaran firmar 
consentimiento informado para ingresar en el estudio. Se excluyeron: pacientes inmunocomprometidos, pacientes con hernia estrangulada o incarcerada, pacientes con hernias recurrentes o pacientes con hernia femoral asociada a hernia inguinal.

Se formaron 2 grupos mediante una tabla de números aleatorios generados por computadora (programa R). Grupo I) pacientes en quienes se utilizó malla de PEBD (mosquitero), monofilamento y trenzado macroporo, con peso de $53,7 \mathrm{~g} / \mathrm{m}^{2}$, con fibras de $0,48 \mathrm{~mm}$ de diámetro, fuerza tensil de $42,7 \mathrm{~N} / \mathrm{cm}$ y poro de $1,8 \mathrm{~mm}^{2} \mathrm{y}$ con un costo total de 5.00 pesos mexicanos por malla de 15 x 15 cm (marca Marlex ${ }^{\circledR}$, Zapopan, México); Grupo II) pacientes en quienes se utilizó malla de PP de la marca Prolene ${ }^{\circledR}$, Ethicon, monofilamento y trenzado macroporo, con peso de $108,1 \mathrm{~g} / \mathrm{m}^{2}$, con fibras de $0,53 \mathrm{~mm}$ de diámetro, fuerza tensil de $156,6 \mathrm{~N} / \mathrm{cm}$, con un poro de $1,6 \mathrm{~mm}^{2}$, con costo de 2.500 pesos mexicanos.

A todos los pacientes se les realizó plastia libre de tensión, ya estandarizada con técnica de Liechtenstein (7), bajo anestesia local, regional o general, entregándose al cirujano la malla correspondiente de acuerdo a la tabla de números aleatorios mencionada. Se administró 30 minutos antes de la incisión, una dosis única de antibiótico de manera profiláctica (cefalotina 1 g por vía intravenosa o ciprofloxacina $400 \mathrm{mg}$, vía oral, en caso que el paciente fuera alérgico a la penicilina). Se fijó la malla con Prolene 2-0. Se prescribió para el control de dolor, ketorolaco $30 \mathrm{mg}$ por via intravenosa, cada 6 horas en el manejo intrahospitalario y 10 mg vía oral cada 6 horas, para el manejo ambulatorio.

Se realizó la evaluación doble ciego del dolor mediante la escala visual del dolor (EVA) y de la presencia de la infección, el rechazo y la recurrencia (tanto por el evaluador, como por el paciente), en citas a la consulta externa a los 7 y 30 días, y vigilancia de rechazo de la malla y recurrencia de la hernia a los 3, 6, y 18 meses posteriores al evento quirúrǵico. Por último, se evaluaron los costos del procedimiento. La concordancia de las mediciones fue evaluada mediante kappa ponderada siendo de 90\% (EVA), 100\% (rechazo) y 100\% (recurrencia). El índice de masa corporal (IMC) se calculó dividiendo el peso sobre la talla al cuadrado.

El presente estudio fue aprobado por los comités de investigación y de ética del Hospital Central Dr. Iǵnacio Morones Prieto; además, se conservó la confidencialidad de los datos.

Se utilizó el programa R versión 3.5.0, para el análisis estadístico. Para la comparación de la frecuencia de infección, recaída o recurrencia entre los grupos, se utilizó $\mathrm{Chi}^{2}$ y t de student o U de Mann Whitney para las variables continuas. Para la comparación del EVA se utilizó regresión logit multinivel, considerando el grupo de estudio como variable predictora y el sexo y la edad como de confusión. Se consideró significativo si $\mathrm{p}<0,05$.

\section{RESULTADOS}

Se estudiaron un total de 31 plastias en 28 pacientes (a 3 pacientes que presentaban hernia inguinal bilateral, se les aleatorizó independientemente cada lado afectado), dividiéndose en 16 plastias con malla de PEBD en el grupo I y 15 plastias con malla de PP en el grupo II; la edad promedio fue de 51,6 años en el primer grupo y de 49,8 años en el segundo. Predominó el sexo masculino en ambos grupos 14/16 en el grupo I y 13/15 en el grupo II. Se registraron 9 pacientes con tabaquismo en el grupo I y 7 en el grupo II y 5 pacientes con otras comorbilidades en cada uno. El IMC promedio fue de $26,64 \mathrm{~kg} /$ $\mathrm{m}^{2}$ en el grupo I y $25,83 \mathrm{~kg} / \mathrm{m}^{2}$ en el II La mayoría de los pacientes se ubicó entre ASA I y II, y solo un paciente del grupo II se clasificó como ASA IV. El tiempo de evolución del padecimiento herniario fue en promedio de 43,8 meses en el primer grupo y 74,3 meses en el segundo.

No se encontraron diferencias estadísticamente significativas en las características 
socio-demográficas estudiadas entre ambos grupos (Tabla I).

Con respecto al dolor post-operatorio inmediato (7 días) en el grupo I fue de 4,7 (rango 1-7) y en el grupo II de 4,6 (rango 0-8). No se reportaron complicaciones. En el post-operatorio tardío, la evaluación del dolor a los 30 días en el primer grupo fue de 2,1 (rango 0-5) y en el segundo de 3,2 (rango 0-7), a los 90 días fue de 0,6 (rango 0-2) y 0,6 (rango 0-2), y a los 180 días de 0,1 (rango 0-1) y 0,3 (rango 0-1) respectivamente. En todas las comparaciones no se encontró diferencia estadísticamente significativa, así como en la frecuencia de uso de analgésicos. Seis pacientes (3 en cada grupo), refirieron parestesias en el sitio de la herida, no incapacitantes y en el 100\% fueron transitorias. No hubo pacientes con inguinodinia crónica.
Ninguno de los pacientes presentó infección del sitio quirúrǵico.

No se observó rechazo de la malla en alguno de los grupos, así como dehiscencias, recurrencias o rechazo a los 18 meses de seguimiento. Un paciente del grupo I, presentó un seroma que no resolvió en la tercera semana, por lo que fue drenado por punción en el consultorio sin complicaciones posteriores.

La estancia hospitalaria fue menor de 24 horas en todos los pacientes (costo de 1 día de estancia hospitalaria en cada caso). Los costos del procedimiento incluyeron para ambos grupos, la realización del procedimiento, que en el hospital donde se realizó este estudio es de 3.500 pesos mexicanos, mas el costo de la malla que es de 2.500 pesos para la de Prolene ${ }^{\circledR}$ y de 5 pesos para la de mosquitero.

TABLA I

CARACTERÍSTICAS SOCIO DEMOGRÁFICAS DE PACIENTES INTERVENIDOS POR HERNIA INGUINAL SIN TENSIÓN POR GRUPO DE ESTUDIO.

\begin{tabular}{|c|c|c|c|}
\hline Caracteristica & $\begin{array}{c}\text { Malla Polietileno } \\
\text { de Baja Densidad } \\
\text { n=16 }\end{array}$ & $\begin{array}{c}\text { Malla } \\
\text { Polipropileno } \\
\mathrm{n}=15\end{array}$ & $\mathrm{p}$ \\
\hline Sexo Masculino & 14 & 13 & NS \\
\hline Edad (años) & $51,6(\mathrm{DE}=14,2)$ & $49,8(\mathrm{DE}=18,1)$ & NS \\
\hline Tabaquismo & 9 & 7 & NS \\
\hline Diabetes Mellitus 2 & 2 & 3 & NS \\
\hline Hipertensión arterial & 3 & 2 & NS \\
\hline Índice de masa corporal $\left(\mathrm{kg} / \mathrm{m}^{2}\right)$ & $26,6(\mathrm{DE}=11,1)$ & $25,8(\mathrm{DE}=9,2)$ & \\
\hline Tempo de evolución previo (años) & $43,8(\mathrm{DE}=19,2)$ & $44,3(\mathrm{DE}=16,8)$ & NS \\
\hline $\begin{array}{l}\mathrm{ASA} \\
\text { a) I } \\
\text { b) II } \\
\text { c) IV }\end{array}$ & $\begin{array}{c}3 \\
13 \\
0\end{array}$ & $\begin{array}{l}5 \\
9 \\
1\end{array}$ & NS \\
\hline $\begin{array}{l}\text { Localización } \\
\text { a) Derecha } \\
\text { b)Izquierda }\end{array}$ & $\begin{array}{l}8 \\
8\end{array}$ & $\begin{array}{l}7 \\
8\end{array}$ & NS \\
\hline $\begin{array}{l}\text { Tipo de Hernia } \\
\text { a) Directa } \\
\text { b) Indirecta } \\
\text { c) Mixta }\end{array}$ & $\begin{array}{c}4 \\
10 \\
2\end{array}$ & $\begin{array}{c}3 \\
10 \\
2\end{array}$ & NS \\
\hline
\end{tabular}

NS $=$ No Significativo

Vol. 59(4): 318 - 324, 2018 


\section{DISCUSIÓN}

Desde que la técnica sin tensión con malla, se convirtió en el procedimiento de elección para el tratamiento de la hernia inguinal (3), se ha buscado la malla ideal, con las siguientes características: inerte, de monofilamento, con poros mayores de $75 \mu$ de diámetro, resistente a la infección, estimulante de la fibroplasia, fácilmente moldeable, que pueda cortarse sin que pierda su integridad estructural, con adaptabilidad a la anatomía de la región inguinal, que no se modifique por los líquidos orgánicos, no ser alergénica ni carcinogénica, que no produzea adherencias intestinales y que sea económica (6). Para satisfacer los criterios mencionados, se han utilizado prótesis de muy variados tipos y que van desde tejidos autólogos, como fascia en sus inicios, hasta materiales de diferentes elementos como plata, alambre, poliéster, polipropileno, vicryl y politetrafluoroetileno expandido (PTFEe); como parte del proceso continuo en la búsqueda de la prótesis idónea $(8,9)$.

En 1962 se desarrolló la malla de polipropileno (Prolene ${ }^{\circledR}$ ), que satisfizo la mayoría de requerimientos de la prótesis ideal (10), aunque uno de los principales inconvenientes de esta prótesis es su costo (11), por lo que en países en vías de desarrollo se ha considerado el empleo de materiales alternativos, algunos inicialmente concebidos y producidos para otros fines comerciales, pero con características similares en la estructura física y química a las prótesis utilizadas en la actualidad, que además han demostrado ser seguras, accesibles e inocuas (12). Se han utilizado materiales, como polipropileno $50 \%$ y polietileno $50 \%$, desde el 2003 por Tongaonkar y col. (5), en 4 centros de la India para la realización de plastias inguinales, sin encontrar rechazos, y con $0,28 \%$ de recidivas e infección, resultados comparables con los obtenidos con mallas comerciales de polipropileno (Prolene ${ }^{\circledR}$ y Marlex®) 5 y a los observados en nuestro estudio, no encontrando recidivas ni otras complicaciones después de un seguimiento de 18 meses.

Freudenberg y col., reportaron en el 2006 un estudio doble ciego, aleatorizado, utilizando mallas de nylon sin encontrar complicaciones graves o infecciones del sitio quirúrgíco (13), al igual que Clarke y col. y Stephenson y col. utilizando mallas de poliéster $(14,15)$. La malla de polietileno de baja densidad (PEBD) inicialmente diseñada como malla protectora contra picadura de mosquito, fue utilizada en la India por Tongaonkar y col. y Kingsnorth y col. desde 2007 con índices de recidivas, infecciones y rechazos, inferiores o iguales a los reportados con las mallas comerciales ya mencionadas $(16,17)$, aun cuando histológicamente, se encuentra respuesta del huésped a cuerpo extraño, con un número significativamente mayor de macrófagos y células inflamatorias a los 14 y 90 días, comparado con la malla de polipropileno, pero con un depósito de fibras de colágeno igual en ambas mallas (18-20). Los presentes resultados, aunque con un tamaño de la muestra menor, concuerdan con lo anterior ya que no se observaron infeceiones en el sitio de la intervención en ninguno de los grupos.

Existe en la literatura experiencia en el uso de malla de PEBD por más de 8 años, en diferentes regiones del mundo, incluyendo otros países en vías de desarrollo, tomando un auge importante en los últimos años (17). No existen datos de haberse utilizado este tipo de material en nuestro medio, por lo que resulta importante dada las posibles diferencias nutricionales, genéticas y sanitarias, que pudieran resultar en un diferente grado de eficacia, aumentando por ejemplo la frecuencia de recidivas o recurrencias. Aportar información en diferentes poblaciones resulta de suma importancia cuando surge una forma alternativa de tratamiento en este caso de plastia inguinal.

Nuestros pacientes presentaron dolor leve en la mitad de los casos, resultados similares a los reportados por Gundre y col. (21). No se observaron infecciones, dehiscencia 
de herida quirúrǵica, rechazos ni recurrencias en ninguno de los grupos, al igual que lo encontrado por Freudenberg y col. (13), donde se reportó $0 \%$ de rechazos e infecciones a diferencia de otros autores que informaron infección grave y profunda del sitio quirúrgico e infecciones superficiales en el $4,7 \%$ de los casos $(5,15)$.

La mayoría de nuestros pacientes fueron ambulatorios y los procedimientos quirúrgiicos se realizaron bajo anestesia regional o local, similar a lo reportado en los estudios anteriores, que traduce menor estancia intrahospitalaria y costos. En nuestro estudio se realizó un seguimiento promedio de $\mathbf{5 4 0}$ días en ambos grupos sin reportar recurrencia, aunque otros investigadores han reportado casos aislados a los 5 años (5).

De acuerdo a lo anterior, consideramos que nuestros resultados sobre los beneficios y seguridad de la malla de polipropileno de baja densidad, utilizada para la reparación de hernia inguinal libre de tensión, concuerdan con lo reportado en otros países, sin que se presenten diferencias significativas en cuanto a la frecuencia de infección, rechazo y recurrencia, cuando se compararon con la malla de polipropileno (Prolene ${ }^{\circledR}$ ); sin embargo, la malla de PEBD es 500 veces más barata que esta última, por lo que es posible considerarla como una alternativa para la plastia inguinal abierta sin tensión, sobre todo en países emergentes como el nuestro.

\section{REFERENCIAS}

1. Prakash D, Heskin L, Doherty S, Galvin $R$. Local anaesthesia versus spinal anaesthesia in inguinal hernia repair: A systematic review and meta-analysis. Surgeon 2017;15(1): 47-57.

2. Guía de práctica clínica. Diagnóstico y tratamiento de hernias inguinales y femorales, CENETEC, Ed., México: Secretaría de Salud; 2008.

3. Amid PK. Lichtenstein tension free hernioplasty: Its inception, evolution, and principles. Hernia 2004; 8(1): 1-7.
4. Spencer NF, Quereshy F, Camilotti BG, Pitzul K, Kwong J, Jackson T, Penner T. Hospital costs associated with laparoscopic and open inguinal herniorrhaphy. J Soc Lap Surg 2014;18(4): e2014.00217.

5. Tongaonkar RR, Reddy BV, Mehta VK, Singh N, Shivade S. Preliminary multicentric trial of cheap indigenous mosquitonet cloth for tension-free hernia repair. Indian J Surg 2003;65:89-95.

6. Kalaba S, Gerhard E, Winder JS, Pauli EM, Haluck RS, Yang J. Design strateǵies and applications of biomaterials and devices for hernia repair. Bioact Mater 2016;1:2-17.

7. Lichtenstein 1, Shulman A, Parviz A. Causas, prevención y tratamiento de la hernia inguinal recurrente. Clínicas Quirúrǵicas de Norte América. 1993;3: 567-583.

8. Paz VL, Atienza MG. Guía de Práctica Clínica de hernia inguinocrural. Santiago de Compostela: Consellería de Sanidade, Axencia de Avaliación de Tecnoloxías Sanitarias de Galicia, avaliat; Serie Avaliación de Tecnologías. Guías de Práctica Clínica: GPC2007/01. 2007.

9. Conze J, Klinge U, Schumpelick V. Hernias. In: Holzheimer RG, Mannick JA, editors. Surgiical Treatment: Evidence-Based and Problem-Oriented. Munich: Zuckschwerdt; 2001.

10. Carbonell TF. Hernia ingúinocrural. Madrid: Ethicon; 2001: Asociación Española de Cirujanos, 2001:19-20.

11. Kingsnorth A, Tongaonkar RR, Awojobi OA. Commentary on: low cost mesh for inguinal hernia repair in resource limited settings. Hernia 2011;15:491-494.

12. Sanders DL. An in vitro study assessing the infection risk of low cost polyethylene mosquito net compared with commercial hernia prosthetics. J Surg Res 2013; (183): E31- E37.

13. Freudenberg S, Sano D, Ouangre E, Weiss C, Wilhelm T. Commercial mesh versus nylon mosquito net for hernia repair: a randomized double blind study in Burkina Faso. World J Surg 2006;30:1784-1789.

14. Clarke MG, Oppong C, Simmermacher R, Park K, Kurzer M, Vanotoo L, Kingsnorth AN. The use of sterilized polyester mosquito net mesh for inguinal hernia repair in Ghana. Hernia 2009;13:155-159. 
15. Stephenson BM, Kingsnorth AN. Safety and sterilization of mosquito net mesh for humanitarian inguinal hernioplasty. World J Surg 2011;35:1957-1960.

16. Stephenson BM, Kingsnorth $\mathrm{AN}$. Inguinal hernioplasty using mosquito net mesh in low income countries: an alternative and cost-effective prosthesis. Br Med J 2011; 343: d7448.

17. Tongaonkar RR. 10 years personal experience of u sing low cost "Low Density Polyethylene (LDPE)" mesh for inguinal hernia repair. Trop Med Surg 2013; 1:136. doi: 10.4172/2329-9088.1000136.

18. Wilhelm TJ, Freudenberg S, Jonas E, Grobholz R, Post S, Kyamanywa P. Sterilized mosquito net versus commercial mesh for hernia repair. An experimental study in goats in Mbarara/Uganda. Eur Surg Res 2007;39:312-317.
19. Sharma M, Sharma DB, Chandrakar SK, Sharma D. Histopathological comparison of mosquito net with polypropylene mesh for hernia repair: An experimental study in rats. Indian J Surg 2015;77(Suppl 2):511514 doi 10.1007/s 12262-013-0904-6.

20. Sanders DL, Kingsnorth AN, Stephenson BM. Mosquito net mesh for abdominal wall hernioplasty: a comparison of material characteristics with commercial prosthetics. World J Surg 2013;37:737-745.

21. Gundre NP, Iyer SP, Subramaniyan P. Prospective randomized controlled study using polyethylene mesh for inguinal hernia mesh plasty as a safe and cost- effective alternative to polypropylene mesh. Updates Surg 2012;64: 37-47. 